\title{
A new target for fetal hemoglobin reactivation
}

\author{
Angela Rivers, ${ }^{1,2}$ Robert Molokie, ${ }^{2,3}$ and Donald Lavelle ${ }^{2,3}$ \\ ${ }^{1}$ Department of Pediatrics, University of Illinois at Chicago; ${ }^{2} J e s s e$ Brown VA Medical Center and ${ }^{3}$ Department of Medicine, University of \\ Illinois at Chicago, Chicago, IL, USA
}

E-mail: DONALD LAVELLE - dlavelle@uic.edu

doi:10.3324/haematol.2019.230904

$I^{\prime}$ nherited hemoglobinopathies, including sickle cell disease (SCD) and the thalassemias, are the most common human monogenic diseases and represent a highly significant global health problem. ${ }^{1,2}$ SCD drastically impacts the quality of life and reduces the life span of approximately 100,000 patients in the US and millions worldwide. Over 300,000 individuals are born with the disease each year with the vast majority in the developing nations of sub Saharan Africa where most of them fail to reach their fifth birthday. Hematopoietic stem cell (HSC) transplantation can be curative, but over $80 \%$ of patients lack a suitable donor. Gene therapy and gene editing technologies also offer the possibility of a cure, but require myeloablative drug-conditioning regimens for successful transplantation of the edited autologous HSC population. The technological and hospital infrastructure required to implement these advanced methods are beyond the resources available in the locations where most patients reside. The only currently available treatment option for patients with $\beta$-thalassemia major is chronic transfusion therapy. Elevated levels of fetal hemoglobin $(\mathrm{HbF} ; \alpha 2 \gamma 2)$ reduce the severity of symptoms and lengthen the life span of SCD patients by inhibiting deoxy HbS polymerization, while in $\beta$-thalassemia patients, increased $\mathrm{HbF}$ alleviates the lack of $\beta$-globin production. Hydroxyurea (HU), a drug approved by the US Food and Drug Administration (FDA) that can increase $\mathrm{HbF}$ in SCD, is not effective in a large subset of patients and, importantly, the increased $\mathrm{HbF}$ is heterogeneously distributed within the erythrocyte population resulting in a large fraction of erythrocytes lacking protective levels. Effective treatment of the large numbers of patients projected worldwide in the coming years would be best accomplished with an affordable, easily-administered, orally-available drug designed to achieve effective increases in $\mathrm{HbF}$ levels. A logical approach to increase $\mathrm{HbF}$ for therapy of the hemoglobinopathies is to intervene with the epigenetic repression mechanism that executes the switch from $\mathrm{HbF}$ to adult hemoglobin ( $\mathrm{HbA}$; $\alpha 2 \gamma 2) .^{3.5}$ In this issue, the Ginder laboratory has identified a specific co-repressor, MBD2-NURD, that is responsible for silencing $\gamma$-globin expression in adult erythroid cells and has delineated critical amino acid residues within the MBD2 protein that recruit the co-repressor containing the epigenetic-modifying enzymes that mediate silencing. ${ }^{6}$ The identification of these sites of recruitment should allow the identification and development of new drugs that interfere with these interactions to alleviate gene repression and increase $\gamma$ globin expression in adult erythroid cells and that, due to the mild phenotype of $\mathrm{MBD} 2^{-/}$mice, ${ }^{7}$ would be expected to have acceptable side-effects in patients.

The switch from $\mathrm{HbF}$ to $\mathrm{HbA}$ expression occurs in late gestation and involves the acquisition of repressive epigenetic marks at the $\gamma$-globin promoter. The first evidence that an epigenetic mechanism might be involved in this switch arose from experimental results showing a correlation between high levels of $\gamma$-globin expression and the lack of DNA methylation in the $5^{\prime} \gamma$-globin promoter region. ${ }^{8}$ Subsequently, high levels of $\mathrm{HbF}$ were observed following treatment of baboons with 5-azacytidine (5-aza), an inhibitor of DNA methylation. ${ }^{9}$ The Ginder laboratory, working in the chicken system, showed that inhibitors of two different epigenetic-modifying enzymes (DNMT1 and HDAC) in combination increased the expression of developmentally silenced globin genes. ${ }^{10}$ Numerous clinical studies have now confirmed the ability of pharmacological DNMT1 inhibitors, (5-aza and decitabine) to increase $\mathrm{HbF}$ in patients with $\beta$-thalassemia and SCD. ${ }^{3}$ In recent years great progress has been made to increase our understanding of the mechanism responsible for developmental $\gamma$-globin silencing by the discovery of three trans-acting, site-specific DNA binding proteins (BCL11A, TR2/TR4, and ZBTB7A) that recognize and bind to specific sequences within the $\gamma$-globin promoter. ${ }^{11,12}$ Critical to the repressive activity of these proteins is their ability to recruit multiprotein co-repressors containing epigenetic-modifying enzymes (DNMT1, HDAC, LSD1, G9A) whose activities directly establish the repressive chromatin environment silencing $\gamma$-globin expression. ${ }^{13,14}$ Pharmacological inhibitors of these enzymes increase $\gamma$-globin expression in various cell culture, mouse, and nonhuman primate model systems, often to impressive levels that would be predicted to provide therapeutic benefits to SCD and $\beta$-thalassemia patients. ${ }^{3-5}$ The major issue hindering use of these drugs in patients are dose-limiting hematologic side-effects that include neutropenia, thrombocytopenia, or thrombophilia.

The methylated DNA binding protein family includes the founding member MeCP2 and at least six additional proteins (MBD1-6) identified by homology searches. MeCP2, MBD1, $\mathrm{MBD} 2$, and $\mathrm{MBD} 3$ each contain a methylated DNA binding domain $(\mathrm{MBD})$ that binds specifically to methylated $\mathrm{CpG}$ residues in vitro. ${ }^{15} \mathrm{MBD} 2$ and $\mathrm{MBD} 3$ are closely related and are $>80 \%$ homologous outside the MBD. ${ }^{16} \mathrm{MBD} 2$ and MBD3 each contain a $C$ terminal coiled-coiled (CC) domain that mediates protein-protein interactions. Additional domains present in MBD2 but absent in MBD3 include an $\mathrm{N}$ terminal glycine-arginine (GR) rich domain, a transcriptional repressor (TRD) domain that overlaps with the $M B D$ and is essential for interaction with the NURD complex, and an intrinsically disordered region (IDR) important for binding to methylated DNA and recruitment of the NURD co-repressor protein complex. Isoforms of $\mathrm{MBD} 2$ lacking one or more of these domains have been identified.

The Ginder laboratory previously identified MBD2 as a repressor of $\gamma$-globin expression through experiments in $\mathrm{MBD}^{-/}$human $\beta$-globin YAC mice ${ }^{17}$ and isolated MBD2 as a component of a purified multi-protein complex that bound the methylated and developmentally silenced $\sigma$-globin gene 
A
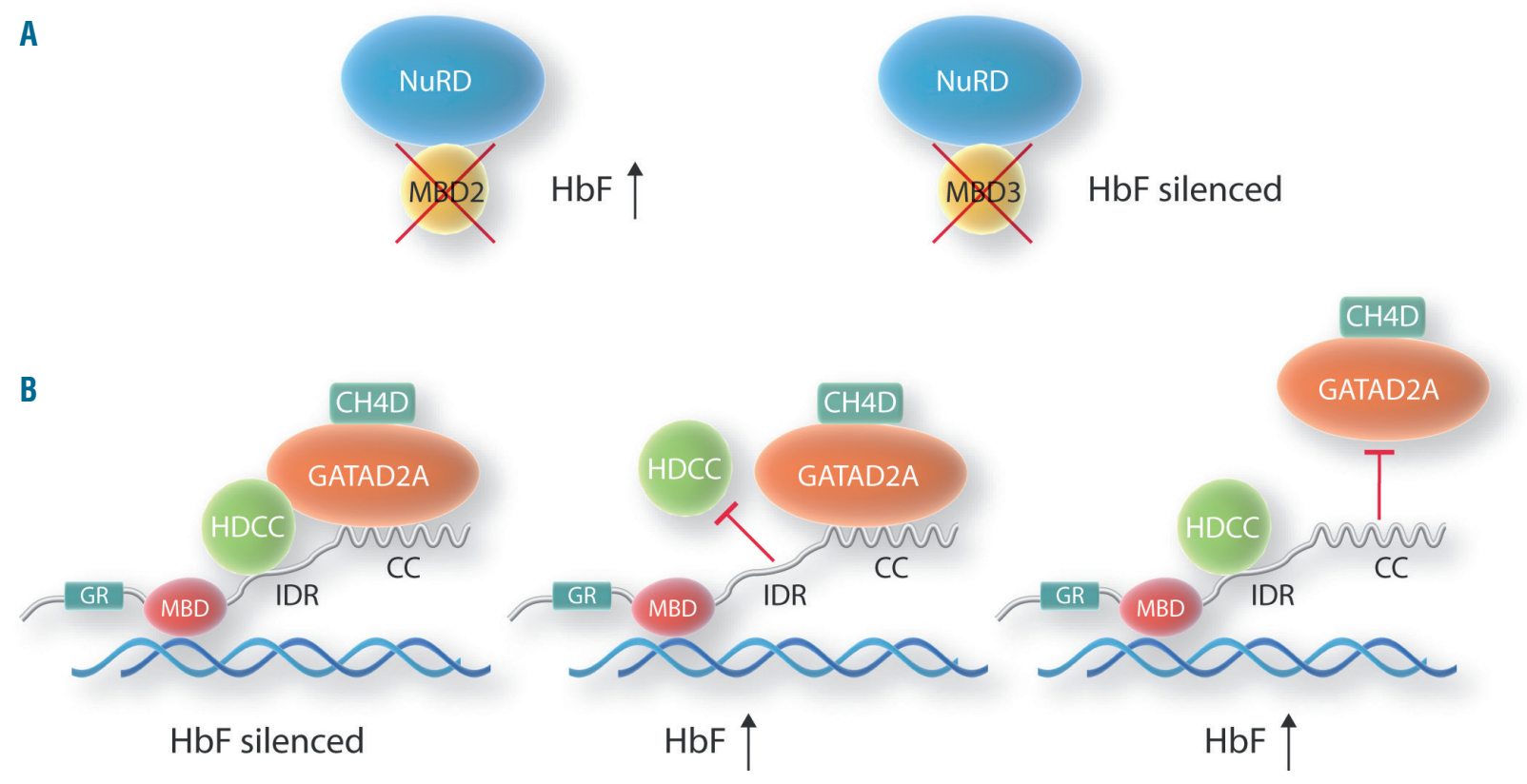

Figure 1. Repression of the $\gamma$-globin gene by MBD2. (A) Contrasting effect of deletions of MBD2 and MBD3 on fetal hemoglobin (HbF). (B) Amino acid substitutions within the intrinsically disordered region (IDR) and coiled-coiled (CC) domains of MBD2 disrupt interactions with components of the NURD co-repressor and fail to repress $\mathrm{HbF}$ in MBD2 knockout cells.

in chicken erythrocytes. ${ }^{18}$ Both $\mathrm{MBD} 2$ and/or MBD3 were identified as components of co-repressor complexes recruited by Bcl11A, ZBTB7A, and TR2/TR4. ${ }^{13,14}$ Although MBD2 and MBD3 were initially thought to be part of the same NURD complex, studies performed in mouse deletion models showed that these proteins have discrete physiological roles as $\mathrm{MBD}^{-/}$mice died in early embryogenesis while $\mathrm{MBD} 2 \%$ mice remained viable and fertile. Subsequent biochemical analyses demonstrated that $\mathrm{MBD} 2$ and $\mathrm{MBD} 3$ were present in separate NURD complexes $^{19}$ thus leading to the question of whether MBD2-NURD and MBD3-NURD have distinct functions in $\gamma$-globin repression. In this issue of the Journal, Yu et al. ${ }^{6}$ has elegantly and clearly answered this question by analysis of $\gamma$-globin expression in biallelic CRISPR/Cas9 knockouts (KO) of MBD2 and MBD3 in the HUDEP-2 human erythroid cell line. $\mathrm{HbF}$ expression was reactivated to high levels in MBD2 KO cells but not in MBD3 KO cells (Figure 1A). Partial knockdown (KD) of MBD2 by lentivirus-delivered siRNA increased $\gamma$-globin expression substantially in both HUDEP-2 and primary human erythroid cell cultures. Importantly, no effect on expression of the known $\gamma$-globin repressors including Bcl11A and ZBTB7A was observed in MBD2 KO cells. Erythroid differentiation was not blocked or substantially altered in MBD2 KO HUDEP2 cells or in MBD2 KD primary erythroid cell cultures. These experiments provide strong and definitive evidence that MBD2 and not MBD3 is responsible for $\gamma$-globin repression in adult human erythroid cells. Recent results of a CRISPR/Cas9 mutagenesis screening have confirmed a specific role for $\mathrm{MBD} 2$ in $\gamma$-globin silencing. ${ }^{20}$ In additional experiments, the role of specific regions of $\mathrm{MBD} 2$ previously shown to function as important sites facilitating protein-protein interactions within co-repressor com- plexes were analyzed for their mechanistic roles in $\gamma$-globin repression (Figure 1B). The effect of amino acid substitutions within the CC domain of MBD2 that mediate interactions with GATAD2A and subsequent recruitment of the chromatin-modifying protein $\mathrm{CH} 4 \mathrm{D}$, and also within the IDR necessary for interaction of MBD2 with an HDAC core complex, were analyzed for their ability to repress $\gamma$-globin expression in $\gamma$-globin expressing MBD2 $\mathrm{KO}$ cells by forced expression of wild-type or mutant $\mathrm{MBD} 2$ delivered via lentiviral vectors. Wild-type MBD2 decreased $\gamma$-globin expression but MBD2 containing sitespecific mutations in the CC domain or IDR did not, indicating that protein-protein interactions facilitated by these regions were critical for $\gamma$-globin repression.

Even though many important questions remain regarding the exact role of MBD2 in $\gamma$-globin silencing, the essential work of identifying and developing small molecule pharmacological agents that target the CC domain and IDR and block the specific contacts mediating the critical functional interactions with other co-repressor proteins can now begin. Because the overall phenotypic effects observed in MBD2 KO mice are minor, it is reasonable to predict that drugs specifically targeting MBD2 would have minimal side effects in patients and thus offer great potential for future therapy for the hemoglobinopathies.

\section{References}

1. Piel FB, Steinberg MH, Rees DC. Sickle Cell Disease. N Engl J Med. 2017:376(16):1561-1573.

2. Modell B, Darlison M. Global epidemiology of haemoglobin disorders and derived service indicators. Bull World health Organ. 2008:86(6):480-487.

3. Ginder GD. Epigenetic regulation of fetal globin gene expression in 
adult erythroid cells. Transl Res. 2015;165(1):115-125

4. Suzuki M, Yamamoto M, Engel JD. Fetal globin gene repressors as drug targets for molecular therapies to treat the $\beta$-globinopathies. Mol Cell Biol. 2014;34(19):3560-3569.

5. Lavelle D, Engel JD, Saunthararajah Y. Fetal hemoglobin induction by epigenetic drugs. Semin Hematol. 2018;55(2):60-67.

6. Yu X, Azzo A, Bilinovich SM, et al. Disruption of the MBD2-NuRD complex but not MBD3-NuRD induces high level HbF expression in human erythroid cells. Haematologica 2019;104(12):2361-2371.

7. Le Guezennec X, Vermeulin M, Brinkman AB, et al. MBD2/NURD and MBD3/NURD, two distinct complexes with different biochemical and functional properties Mol Cell Biol. 2006;26(3):843-851.

8. Van der Ploeg LH, Flavell RA. DNA methylation in the human gamma delta beta-globin locus in erythroid and nonerythroid tissues. Cell. 1980;19(4):947-958.

9. DeSimone J, Heller P, Hall L, Zwiers D. 5-azacytidine stimulates fetal hemoglobin synthesis in anemic baboons. Proc Natl Acad Sci U S A. 1982;79(14):4428-4431.

10. Ginder GD, Whitters MJ, Pohlman JK. Activation of a chicken embryonic globin gene in adult erythroid cells by 5 -azacytidine and sodium butyrate. Proc Natl Acad Sci USA. 1984;81(13):3954-3958.

11. Vinjamur DS, Bauer DE, Orkin SH. Recent progress in understanding and manipulating haemoglobin witching for the haemoglobinopathies. Br J Haematol. 2018;180(5):630-643.

12. Wienert B, Martyn GE, Funnell APW, Quinlan KGR, Crossley M. Wake-up sleepy gene: Reactivating fetal globin for $\beta$-hemoglobinopathies. Trends Genet. 2018;34(12):927-940.
13. Cui S, Kolodziej KE, Obara N, et al. Nuclear receptors TR2 and TR4rcruit multiple epigenetic transcriptional corepressors that associate specifically with the embryonic $\beta$-type globin promoters in differentiated adult erythroid cells. Mol Cell Biol. 2011;31(16):32983311

14. Xu J, Bauer DE, Kerenyi MA, et al. Corepressor-dependent silencing of fetal hemoglobin expression by BCL11A. Proc Natl Acad Sci U S A. 2013;110(16):6518-6523.

15. Du Q, Luu P-L, Stirzaker C, Clark SJ. Methyl-CpG-binding domain proteins:readers of the epigenome. Epigenomics. 2015;7(6):10511073

16. Menafra R, Stunnenberg HG. MBD2 and MBD3: elusive functions and mechanisms. Front Genet. 2014;5:428.

17. Rupon JW, Wang SZ, Gaensler K, Lloyd J, Ginder GD. Methyl binding domain protein 2 mediates gamma-globin gene silencing in adult human betaYAC transgenic mice. Proc Natl Acad Sci U S A. 2006;103(17):6617-6622

18. Kransdorf EP, Wang SZ, Zhu SZ, Langston TB, Rupon JW, Ginder GD. MBD2 is a critical component of a methyl cytosine-binding protein complex isolated from primary erythroid cells. Blood. 2006;108(8):2836-2845.

19. Hendrich B, Guy J, Ramsahoye B, Wilson VA, Bird A. Closely related proteins MBD2 and MBD3 play distinctive but interacting roles in mouse development. Genes Dev. 2001;15(6):710-723.

20. Sher F, Hossain M, Seruggia D, et al. Rational targeting of a NuRD subcomplex guided by comprehensive in situ mutagenesis. Nat Genet. 2019;51(7):1149-1159.

\title{
Are we ready to use precision medicine in chronic myeloid leukemia practice?
}

\author{
Dennis Dong Hwan Kim
}

Leukemia Program, Department of Medical Oncology and Hematology, Princess Margraret Cancer Centre, University Health Network, University of Toronto, Toronto, ON, Canada

E-mail: DENNIS DONG HWAN KIM - dr.dennis.kim@uhn.ca

doi:10.3324/haematol.2019.231753

○ ver the last two decades, the introduction of tyrosine kinase inhibitors (TKI) and advances in $B C R$ $A B L 1$ monitoring using quantitative polymerase chain reaction (qPCR) have significantly improved treatment outcomes in chronic myeloid leukemia (CML) patients. ${ }^{1}$ Not only the introduction of TKI increased the life expectancy of CML patients $(98 \%$ of age-matched healthy control), but also the incorporation of $B C R-A B L 1$ monitoring using qPCR significantly improved outcomes of CML patients by identifying those cases developing TKI failure and progressing to the advanced phase ${ }^{2,3}$ However, it is still challenging to predict patients at high risk for TKI failure at initial diagnosis of CML before commencing TKI therapy. Thus, major challenges still remain, including lack of accurate risk stratification at initial diagnosis.

The current algorithm for CML management is mainly based on monitoring $B C R-A B L 1$ using $\mathrm{qPCR} .{ }^{3}$ Despite its good performance, there are still remaining issues some of which include: i) how to select upfront TKI drug in a newly diagnosed CML patient (imatinib vs. newer generation TKI); ii) how to switch TKI therapy in a patient who developed TKI resistance, but without ABL1 kinase domain mutations; and iii) how to predict which patients are at high risk of progression to blastic crisis. Thus, there is an urgent demand for novel biomarkers in managing CML beyond monitoring $B C R-A B L 1$ fusion transcripts. Given this, how can we go forward from here?
Let us look back at routine CML practice 20 years ago when TKI therapy and qPCR-based $B C R$ - $A B L 1$ monitoring were not available. ${ }^{4}$ When a patient was newly diagnosed with chronic phase CML, the first step would be the identification of an HLA-matched donor for allogeneic hematopoietic cell transplantation (HCT) and co-ordination of allogeneic HCT within two years from initial diagnosis before the patient progressed to advanced phase. If an appropriate donor was not available, interferon therapy was a treatment of choice. Disease monitoring was mainly based on the metaphase cytogenetic test for which bone marrow aspiration should be performed every 6 months to assess cytogenetic response. Let us compare it with current CML practice, which has changed significantly over the last two decades. First, we no longer initiate a search for an HLA-matched donor search until TKI failure or intolerance to more than two TKI is suspected. ${ }^{3}$ Bone marrow examination does not need to be repeated as frequent as $B C R$ $A B L 1$ qPCR on peripheral blood which is the mainstay of disease monitoring. So, what will happen in the future? CML practice will evolve and will be transformed again from the current routine practice. However, what we do not know yet is how this will be achieved and what changes will be applied.

Precision medicine is becoming the mainstream of future medicine. It has been implemented in the clinical practice in acute myeloid leukemia (AML), ${ }^{5}$ and myeloproliferative 